\title{
Haplo-identical Haematopoietic Stem Cell Transplantation in Refractory Hodgkin Lymphoma After Carmustine, Etoposide, Cytarabine, Melphalan Conditioning Regimen
}

\author{
Nour B Abdeljelil, 1,2 Dorra Belloumi, 1,2 Sabrine Mekni, ${ }^{1,2}$ Amel Lakhal, ${ }^{1,2}$ Lamia Torjemane, ${ }^{1,2}$ Saloua Ladeb, ${ }^{1,2}$ \\ Tarek B Othman ${ }^{1,2}$ \\ 1. Department of Haematology, National Bone Marrow Transplantation Centre of Tunis, Tunis, Tunisia; 2. Faculté de Médecine de Tunis, \\ Université de Tunis El Manar, Tunis, Tunisia
} lymphoma (HL) or HL relapsing after autologous peripheral blood stem cell transplantation (PBSCT). Haplo-identical HSCT (haploHSCT) is an alternative option for these patients when a human leukocyte antigen (HLA)-identical sibling donor is not available. We report the case of an 18-year-old man who underwent a haplo-HSCT for early relapsed stage IV HL. He was scheduled for autologous PBSCT and received a conditioning regimen with carmustine, etoposide, cytarabine and melphalan (BEAM). On Day 0, the graft was damaged at thawing time, and he underwent a haplo-HSCT because he had no HLA-identical sibling donor. This represents the first case of a haplo-HSCT after the BEAM conditioning regimen. We describe the feasibility and success of upfront haplo-HSCT in this setting.

\section{Keywords}

Hodgkin lymphoma, haplo-identical stem cell transplantation, conditioning regimen, stem cells, post-transplant cyclophosphamide, complications

Disclosure: Nour B Abdeljelil, Dorra Belloumi, Sabrine Mekni, Amel Lakhal, Lamia Torjemane, Saloua Ladeb and Tarek B Othman have no financial or non-financial relationships or activities to declare in relation to this article.

Review process: Double-blind peer review.

Compliance with ethics: All procedures were followed in accordance with the responsible committee on human experimentation and with the Helsinki Declaration of 1975 and subsequent revisions, and informed consent was received from the patient involved in this case study. Authorship: The named authors meet the International Committee of Medical Journal Editors (ICMJE) criteria for authorship of this manuscript, take responsibility for the integrity of the work as a whole, and have given final approval for the version to be published.

Access: This article is freely accessible at touchONCOLOGY.com (c) Touch Medical Media 2021

Received: 3 July 2021

Accepted: 29 July 2021

Published online: 5 October 2021

Citation: touchREVIEWS in Oncology \& Haematology. 2021;17(2):111-3

Corresponding author: Nour Ben Abdeljelil, Centre National de Greffe de la Moelle Osseuse, Rue Jebel Lakhdar, 1006 Bab Saadoun, Tunis, Tunisia. E: nour.abdejli@gmail.com

Support: No funding was received for the publication of this article.
Allogeneic haematopoietic stem cell transplant (allo-HSCT) remains a potential curative therapeutic option for patients with refractory/relapsed Hodgkin lymphoma (HL) in whom an autologous peripheral blood stem-cell transplantation (PBSCT) has failed. This is explained by the additional benefit of a graft-versus-lymphoma (GVL) effect. Unfortunately, HLA-identical donors are not available for all patients. In such cases, haplo-identical HSCT (haplo-HSCT) with post-transplant cyclophosphamide could be a therapeutic option. The advantages of this transplant are the easy donor availability and instant access to the donor, however, infectious complications remain the major problem of haplo-HSCT due to delayed immune reconstitution.

\section{Diagnosis and previous treatment}

A 18-year-old man was diagnosed in September 2018 with stage III, nodular, sclerosing, classical $\mathrm{HL}$ that expressed CD30+, CD15+. At diagnosis, he had enlarged left supraclavicular lymph nodes, and positron emission tomography-computed tomography (PET-CT) showed supra- and infradiaphragmatic enlarged lymph nodes with a mediastinal mass measuring $55 \times 69 \mathrm{~mm}$. The patient received two cycles of escalated BEACOPP (bleomycin, etoposide, adriamycin, cyclophosphamide, vincristine, procarbazine and prednisone) after which complete metabolic remission (CMR) was achieved. Thus, the chemotherapy was de-escalated to adriamycin, bleomycin, vincristine and dacarbazine (ABVD). After four cycles of ABVD, the patient maintained CMR with a persistent, residual, anterosuperior mediastinal mass measuring $57 \times 23 \mathrm{~mm}$, which was irradiated at $30 \mathrm{~Gy}$.

Nine months after completing radiotherapy, the $\mathrm{HL}$ relapsed with stage IV bone involvement. The PET-CT showed metabolic activity in the supra- and infra-diaphragmatic lymph nodes, and revealed new bone lesions in the left clavicle and in the fourth lumbar vertebra. The residual mediastinal mass had decreased in size with no evidence of hypermetabolism. Documented histological relapse was not carried out. The patient was treated with three cycles of ifosfamide, gemcitabine and vinorelbin (IGEV) and achieved a second CMR. He was referred to our centre for autologous PBSCT and received BEAM conditioning. On Day 0 of autologous PBSCT, the graft was damaged at thawing time, necessitating an urgent allo-HSCT from a human leukocyte antigen (HLA)-identical sibling donor, which was the only type of allo-HSCT performed in our centre. In the absence of a geno-identical donor, haplo-HSCT was the only rapid alternative for this patient.

\section{Transplantation}

The donor was the patient's mother, a 55-year-old woman with four out of eight HLA matches with her son. Both the donor and the recipient had negative anti-HLA antibodies and negative serology for hepatitis C virus and HIV. The donor had negative hepatitis B virus (HBV) serology, 
and the patient's vaccine induced HB surface antibodies. The donor and recipient were positive for cytomegalovirus (CMV) immunoglobulin (Ig)G. Donor-specific anti-HLA antibody screening was negative in the patient. ABO-compatible, unmanipulated PBSC allograft was infused 19 days after the start of BEAM conditioning, with infusion of $3.52 \times 10^{6}$ CD34 cells/kg. Graft-versus-host disease (GVHD) prophylaxis consisted of mycophenolate mofetil (MMF) and cyclosporine starting from Day 5 post-haplo-HSCT ( $3 \mathrm{~g} /$ day and $3 \mathrm{mg} / \mathrm{kg} /$ day, respectively) and posttransplant cyclophosphamide at Days 3 and 4 ( $50 \mathrm{mg} / \mathrm{kg} /$ day $)$. Anti-viral prophylaxis was assured by intravenous acyclovir. The patient had been in the aplastic phase since Day 9 post-BEAM.

Fever occurred within a few hours of the infusion of graft and remained for 5 days without documented infection. On Day 23 post-haplo-HSCT, the patient achieved neutrophil recovery with an absolute neutrophil count of 520/uL. Complete donor chimerism was confirmed by quantitative polymerase chain reaction (qPCR) on Day 33 . On Day 12 , he developed grade 2 acute gastrointestinal GVHD, which was corticosensitive. On Day 27, he developed posterior reversible encephalopathy syndrome (PRES) with headache, confusion and generalized tonic-clonic seizures with concomitant hypertension. Cerebral magnetic resonance imaging demonstrated cortical and subcortical posterior parieto-occipital signal abnormalities, T1 hypointense and T2/FLAIR hyperintense. Symptoms resolved after withdrawal of cyclosporine and initiation of benzodiazepines.

On Day 40, CMV was detected by qPCR in blood (viral load $<150$ copies/ $\mathrm{mL}$ ). Pre-emptive therapy with foscavir ( $90 \mathrm{mg} / \mathrm{kg}$ twice daily) was started. Serum CMV viral load increased after 10 days of anti-viral therapy. The patient subsequently developed a grade 3 haemorrhagic cystitis on Day 45 , without detection of BK virus in blood. Foscavir was stopped and switched to cidofovir. The hyperhydration, bladder irrigation and reduced immunosuppressive treatment led to regression of haematuria, and macroscopic haematuria disappeared on Day 149. The MMF was stopped on Day 54 , steroids were quickly tapered and cyclosporine was resumed on Day 44 to avoid GVHD reactivation. Serum CMV viral load continued to increase $(11,700$ copies $/ \mathrm{mL})$ after the second dose of cidofovir, which was stopped. Patient was treated then with ganciclovir + foscavir. Serum CMV viral load decreased to 150 copies/mL within 2 weeks, and became negative on Day 115 post-transplantation. On Day 99, he developed invasive sinus and pulmonary aspergillosis. The nose swab culture revealed Aspergillus niger that was susceptible to voriconazole. He was treated with voriconazole with a good clinical response. Steroids were stopped on Day 112 post-haplo-HSCT. With a follow-up of 8.5 months, he is still in CMR. He did not develop chronic GVHD.

\section{Discussion}

Autologous PBSCT is the standard salvage therapy for relapsed/refractory $\mathrm{HL}$. Allogeneic HSCT provides prolonged complete remissions in posttransplant, relapsed/refractory $\mathrm{HL}$; however, it is generally a last resort due to the high risk of non-relapse mortality. Haplo-HSCT is a treatment option for these patients in the absence of an HLA-identical sibling donor. ${ }^{1}$ For logistical reasons, we do not perform this type of transplant in our department. We report the case of patient with early relapsed HL with stage IV bone involvement. After achieving a second CMR, he was a candidate for autologous PBSCT and received BEAM conditioning, but the graft was damaged at thawing time. We therefore performed a haploHSCT because he had no HLA-identical sibling donor. To our knowledge, this is the first case of a haplo-HSCT after BEAM conditioning in HL. In fact, the most widely used conditioning regimen in haplo-HSCT for HL is that developed by Baltimore team. ${ }^{1}$ It is a reduced intensity conditioning
(RIC) combining fludarabine (150 mg/m²), cyclophosphamide (29 mg/ $\mathrm{kg}$ ) and total body irradiation (TBI) at $2 \mathrm{~Gy}$. The Lymphoma Working Party (LWP)-European Society for Blood and Marrow Transplantation (EBMT) had recently summarized data of haplo-HSCT using post-transplant cyclophosphamide in advanced $\mathrm{HL}$. They reported a 1-year non-relapse mortality of around $20 \%$, a 2-year cumulative incidence of relapse of $24-40 \%$, a 2 -year progression-free survival of around $50 \%$ and a 2-year overall survival of $58-67 \% .^{2}$

We used a PBSC graft to enhance chances of rapid engraftment. The reported source of stem cells in haplo-HSCT is mainly bone marrow. Few studies have reported outcomes of PBSCT after a myeloablative conditioning (MAC) regimen, ${ }^{3-6}$ and there are no randomized studies comparing use of bone marrow with use of PBSC graft after MAC in haplo-HSCT. Two retrospective studies comparing bone marrow and PBSC were carried out after RIC and showed no differences in terms of acute and chronic GVHD, speed of immune reconstitution, platelet reconstitution and non-relapse mortality. ${ }^{5.7}$ However, the EBMT group and Center for International Blood and Marrow Transplant Research showed increased acute GVHD grades $2-4$ with PBSC grafts compared with bone marrow after unmanipulated haplo-HSCT in patients conditioned with MAC or RIC. ${ }^{8,9}$

In our case, the patient developed grade 2, gastrointestinal, acute GVHD that responded to steroids. The combination of female donor and male recipient increases the risk of acute GVHD in haplo-HSCT. ${ }^{10,11}$ This risk is further increased in haplo-identical transplantation with a mother donor." The rates of acute GVHD grades $2-4$ seem to be higher in studies using PBSC than in bone marrow grafts after MAC, with no significantly increased non-relapse mortality, ${ }^{12,13}$ suggesting manageable GVHD. ${ }^{14}$

Bacigalupo et al. reported that post-transplant cyclophosphamide given on Days 3 and 5 results in a lower risk of grades $3-4$ acute GVHD in young patients grafted from young donors than in older patients grafted from older donors (3\% versus $8 \%$, respectively). ${ }^{15}$ In the same perspective, Ruggeri et al. showed that starting immunosuppression therapy (cyclosporine A + MMF) early at Day 0 or Day 1, with post-transplant cyclophosphamide administered on Days 3 and 5 , reduces the incidence of grade II-IV acute GVHD and improves refined GVHD-free, relapse-free survival (rGRFS). ${ }^{16}$

One of the drawbacks of haplo-HSCT is the high incidence of opportunistic infections, especially viral infections, due to delayed immune reconstitution. ${ }^{17}$ Our patient developed invasive aspergillosis and refractory CMV infection with a rapid rise in viral load despite appropriate treatment. Foscavir was the initial treatment because of thrombocytopenia and neutropenia. The CMV viral load remained detectable for 30 days and declined with ganciclovir + foscavir. The profound immunosuppression caused by steroid use and the considerable dose of alkylating agents (melphalan and cyclophosphamide) likely led to the persistence of the CMV infection and to the occurrence of aspergillosis. ${ }^{18}$

He also developed non-BK virus haemorrhagic cystitis. Mori et al. reported that BK virus haemorrhagic cystitis was more frequently observed in patients with excessive immune reactions, such as GVHD, pre-engraftment immune reaction and haemophagocytic syndrome, whereas immune deficiency is associated with adenovirus haemorrhagic cystitis. ${ }^{19}$ In our patient, adenovirus could not be excluded because of a shortage of laboratory kits for adenovirus PCR. The incidences of CMV infection and viral haemorrhagic cystitis were reported to be higher 
when post-transplant cyclophosphamide was used in haplo-HSCT compared with HSCT with matched related donors and with voluntary unrelated donors. ${ }^{20}$

High-dose alkylating agents, chemotherapy and radiotherapy received before autologous PBSCT may also potentiate the risks of myelodysplastic syndrome and secondary cancers in this patient. ${ }^{21,22}$

Our patient developed PRES and hypertension while using steroids for acute GVHD. The incidence of PRES in haplo-HSCT has been rarely reported. The largest retrospective study conducted by Qi Chen et al. ${ }^{23}$ in 180 patients receiving haplo-HSCT reported a PRES incidence of $1.17 \%$, which was significantly lower than reported in allo-HSCT with HLAidentical sibling donors. ${ }^{24-26}$ Grades 2-4 acute GVHD and hypertension were identified as risk factors for developing PRES after haplo-HSCT. ${ }^{24}$

Cyclosporine was reintroduced despite the PRES, because the corticosteroids were rapidly tapered to overcome persistent CMV reactivation and because of the unavailability of mechanistic target of rapamycin (mTOR) inhibitors. Resuming cyclosporine allowed the control of GVHD without recurrence of neurotoxicity. Management of GVHD in the context of cyclosporine-related neurotoxicity constitutes a real challenge. According to Straathof et al., ${ }^{27}$ re-introduction of calcineurin inhibitors (Cl) after $\mathrm{Cl}$-induced neurotoxicity was tolerated in around half of patients and could be a viable option. ${ }^{27,28}$ Chohan et al. reported recurrence of neurotoxicity in $41 \%$ of patients after resuming $\mathrm{Cls}$, and the neurological presentation was identical to that of the first episode of neurotoxicity. ${ }^{28}$

To our knowledge, we report the first case of relapsed $\mathrm{HL}$ treated by an upfront and urgent haplo-HSCT after salvage therapy and BEAM conditioning. The management of serious infections caused by delayed immune reconstitution has been a major challenge in this setting. Reduction of post-transplant cyclophosphamide doses, considering the high-dose of melphalan previously received during BEAM conditioning, would have helped to reduce infectious complications post-haplo-HSCT in this patient.
1. Luznik L, O'Donnell PV, Symons HJ, et al. HLA-haploidentical bone marrow transplantation for hematologic malignancies using nonmyeloablative conditioning and high-dose, posttransplantation cyclophosphamide. Biol. Blood Marrow Transplant. 2008;14;641-50

2. Dietrich $\mathrm{S}$, Dreger $\mathrm{P}$, Hermine $\mathrm{O}$, et al. Haploidentical stem cell transplantation for patients with lymphoma: a position statement from the Lymphoma Working Party-European Society for Blood and Marrow Transplantation. Bone Marrow Transplant. 2020;55:317-24.

3. Raiola AM, Dominietto A, Ghiso A, et al. Unmanipulated haploidentical bone marrow transplantation and posttransplantation cyclophosphamide for hematologic posttransplantation cyclophosphamide for hematologic
malignancies after myeloablative conditioning. Biol Blood malignancies after myeloablative condi

4. Burroughs LM, O'Donnell PV, Sandmaier BM, et al. Comparison of outcomes of HLA-matched related, unrelated, or HLAhaploidentical related hematopoietic cell transplantation following nonmyeloablative conditioning for relapsed or refractory Hodgkin lymphoma. Biol Blood Marrow Transplant. 2008;14:1279-87

5. Bradstock K, Bilmon I, Kwan J, et al. Influence of stem cell source on outcomes of allogeneic reduced-intensity conditioning therapy transplants using haploidentical related donors. Biol Blood Marrow Transplant. 2015;21:1641-45.

6. Cieri N, Greco R, Crucitti L, et al. Post-transplantation cyclophosphamide and sirolimus after haploidentical hematopoietic stem cell transplantation using a treosulfanbased myeloablative conditioning and peripheral blood stem based myeloablative conditioning and peripheral blood
cells. Biol Blood Marrow Transplant. 2015;21:1506-14.

7. Cells. Biol Blood Marrow Transplant. 2015;21:1506-14.

Castagna $L$, Crocchiolo R, Furst S, et al. Bone marrow
compared with peripheral blood stem cells for haploidentical transplantation with a nonmyeloablative conditioning regimen and post-transplantation cyclophosphamide. Biol Blood Marrow Transplant. 2014;20:724-9.

8. Ruggeri A, Labopin M, Bacigalupo A, et al. Bone marrow versus mobilized peripheral blood stem cells in haploidentical transplants using posttransplantation cyclophosphamide. Cancer. 2018;124:1428-37.

9. Bashey A, Zhang M-J, McCurdy SR, et al. Mobilized peripheral blood stem cells versus unstimulated bone marrow as a graft source for T-cell-replete haploidentical donor transplantation using post-transplant cyclophosphamide. I Clin Oncol. 2017;35:3002-9.

10. Kasamon YL, Luznik L, Leffell MS, et al. Nonmyeloablative HLA-haploidentical bone marrow transplantation with high-dose posttransplantation cyclophosphamide: effect of HLA disparity on outcome. Biol Blood Marrow Transplant 2010;16:482-9

11. Piemontese S, Ciceri F, Labopin M, et al. A survey on unmanipulated haploidentical hematopoietic stem cell transplantation in adults with acute leukemia. Leukemia. 2015;29:1069-75

12. Solomon SR, Sizemore CA, Sanacore M, et al. TBI-based myeloablative haploidentical stem cell transplantation is a safe and effective alternative to unrelated donor transplantation in patients without matched sibling donors. Biol Blood Marrow Transplant. 2015;21:1299-307.

13. Bashey $A$, Zhang $X$, Jackson $K$, et al. Comparison of outcomes of hematopoietic cell transplants from T-replete haploidentical donors using post-transplantation cyclophosphamide with 10 of 10 HLA-A, - B, -C, -DRB1, and -DQB1 allele-matched unrelated donors and HLA-identical sibling donors: A multivariable analysis including disease risk index. Biol Blood Marrow Transplant. 2016;22:125-33.

14. Nguyen $S$, Chalandon $Y$, Lemarie $C$, et al. Greffe de cellules-souches hématopoiétiques haplo-identiques: recommandations de la Société francophone de greffe de moelle et de thérapie cellulaire (SFGM-TC). Bull Cancer (Paris). 2016;103:S229-42.

15. Bacigalupo A, Raiola AM, Dominietto A, et al. Graft versus host disease in unmanipulated haploidentical marrow transplantation with a modified post-transplant cyclophosphamide (PT-CY) regimen: an update on 425 patients. Bone Marrow Transplant. 2019;54:708-12.

16. Ruggeri A, Labopin M, Battipaglia G, et al. Timing of post-transplantation cyclophosphamide administration in haploidentical transplantation: A comparative study on behalf of the Acute Leukemia Working Party of the European Society for Blood and Marrow Transplantation. Biol Blood Marrow Transplant. 2020;26:1915-22.

17. Spitzer TR. Haploidentical stem cell transplantation: the always present but overlooked donor. Hematology Am Soc Hematol Educ Program. 2005:390-395

18. Lionakis MS, Kontoyiannis DP. Glucocorticoids and invasive fungal infections. Lancet. 2003;362:1828-38.

19. Mori Y, Miyamoto T, Kato K, et al. Different risk factors related to adenovirus- or BK virus-associated hemorrhagic cystitis following allogeneic stem cell transplantation. Biol Blood Marrow Transplant. 2012;18:458-65.

20. García-Cadenas I, Esquirol A, Bosch-Vilaseca A, et al. Patterns of infection and infectious-related mortality in patients receiving post-transplant high dose cyclophosphamide as receiving post-transplant high dose cyclophosphamide as
graft-versus-host-disease prophylaxis: impact of HLA donor graft-versus-host-disease prophylaxis: impact of HLA
matching. Bone Marrow Transplant. 2020;56:818-27.

21. Vega-Stromberg T. Chemotherapy-induced secondary malignancies. J Infus Nurs. 2003;26:353-61.

22. Green DM, Hyland A. Barcos MP, et al. Second malignant neoplasms after treatment for Hodgkin's disease in childhood or adolescence. J Clin Oncol. 2016;18:1492-9.

23. Chen Q, Zhao X, Fu H-X, et al. Posterior reversible encephalopathy syndrome (PRES) after haploidentical haematopoietic stem cell transplantation: incidence, risk factors and outcomes. Bone Marrow Transplant. 2020;55:203542.

24. Gaziev J, Marziali S, Paciaroni K, et al. Posterior reversible encephalopathy syndrome after hematopoietic cell transplantation in children with hemoglobinopathies. Biol Blood Marrow Transplant. 2017:23:1531-40.

25. Tavares M, Arantes M, Chacim S, et al. Posterior reversible encephalopathy syndrome in children with hematologic malignancies. J Child Neurol. 2015;30:1669-75.

26. Siegal D, Keller A, Xu W, et al. Central nervous system complications after allogeneic hematopoietic stem cell transplantation: incidence, manifestations, and clinical significance. Biol Blood Marrow Transplant. 2007;13:1369-79.

27. Straathof $K$, Anoop P, Allwood Z, et al. Long-term outcome following cyclosporine-related neurotoxicity in paediatric allogeneic haematopoietic stem cell transplantation. Bone Marrow Transplant. 2017;52:159-62

28. Chohan R, Vij R, Adkins $D$, et al. Long-term outcomes of allogeneic stem cell transplant recipients after calcineurin inhibitor-induced neurotoxicity. Br J Haematol. 2003;123:110-3. 